\title{
LI. Memoir upon the decomposition of water, and of the bodies which it holds in solution, by means of galvanic electricity
}

\section{I. T. de Grotthius}

To cite this article: C. I. T. de Grotthius (1806) LI. Memoir upon the decomposition of water, and of the bodies which it holds in solution, by means of galvanic electricity, Philosophical Magazine Series 1, 25:100, 330-339, DOI: 10.1080/14786440608563457

To link to this article: http://dx.doi.org/10.1080/14786440608563457

曲 Published online: 18 May 2009.

Submit your article to this journal $\pi$

Џll Article views: 5

Q View related articles $\llbracket$ 
L1. Memoir * upon the Decomposition of Water, and of the Bodies which it holds in Solution, by means of Galvanic Electricity. By C. I. T. DE Grotruius t.

\section{Chap. I.}

Action of Galvanic Electricity upon certuin Bodies dissolved in Water.

I. W Irnour wasting time on the discussion of the multitude of imaginary bypotheses invented to explain the decomposition of water by the electrometer apparatus, I shall give a general theory of the decomposition of liquids by Galvanic electricity, which, in my opinion, brings the effects of the latter to a simple and satisfactory explanation. I was led to this theory by the following observations:

II. When a current of Galvanic electricity is made to pass through a saturated metallic solution, the intensity of this current being proportioned to the interval occupicd by the liquid, and comprised between the extremities of the two conductor wires, interesting phænomena are discovered even by an observer who does not trouble himself to investigate the cause. At the extremity of the wire in contact with the disk of zinc, oxygen is disengaged; while at the extremity of the wire in contact with the disk of copper, the molecules of the metal in solution are revived, assuming a symmetrical arrangement, which extends in the direction of the Galvanic current.

III. This arrangement is nothing else than an imperfect crystallization of metallic molecules, exactly similar to that known by the name of arborisation, and which takes place upon precipitating metals in solution by other metals. The old chemists added to the word arbor the name of the deity to whom the metal was consecrated. Thence come the old names of arbor Diance, arbor Martis, arbor Veneris, \&c. Of all the phænomena presented to us by Galvanism, no one

* From Annales de Chimie, tom. lviii. p. 54.

$\uparrow$ This memoir was printed at Rome in 1805. We presume the perusal of it here will give pleasure to our readers, as the author himself has also rejuested ue to reprint it.-Note of French editor. 
is so fine or so interesting as this vegetation, presenting to our view the image of a fine shrub, furnished with its foliage and adorned with the most beautiful metallic brilliance.

IV. Wollaston, the celebrated English chemist, has already noticed, that upon establishing a current of electricity in the solution of a metal, the latter is revived at the extremity of the conductor endued with negative electricity; but I am ignorant whether or no he also perceived that this revival is susceptible of assuming a symmetrical arrangement, when the action has enough. of energy and has lasted a sufficient time.

V. All the metals in solution are not equally decomposed by Galvanic electricity. From nitrate of manganese I obtained gaseous bubbles at the negative pole* in place of a metallic depusit; and it seems that when in similar circumstances, the metal, in solution has more affinity for oxygen than bydrogen has for this principle, it is the water which alone suffers the decomposition.

VI. During the arborisation of the metals at the negativo pole, no gas is seen to be disengaged; whence I conclude, either that the hydrogen arising is combined with the oxygen of the metallic nxide, or that the action is only exercised upon this oxide and not upon the water. This last conclusion ought to be a true one; for we can scarcely admit that the bydrogen is able to carry off completely the oxygen from the oxides of zine and iron, as well as from certain acids their solvents, in which these two metals are not dissolved, except after having produced an effect contrary to this admission, by decomposing water.

VII. Of all the metallic salts which I submitted to the action of the electrometer apparatus, the acetate of lead and the muriate of tin $\uparrow$ presented the most beautiful vegetation.

- I shall in future make use of the phrase positive pole to express the extremity of the wire communicating with the disk of zinc, and of the term negative pole to express the extramity of the wire in contact with the disk of copper.

+1 have also ubtained effects more or less remarkable from the nitro-muriates of gold and platina, frcm the nitrates of zinc, copper, mercury, and cobalt; from the sulphates of zinc and iron, from the stannite of muriated potash, and from muriate of iron.

That 
That of lead imitates the appearance of fern leaves; and upon the ramifications of tin I have often seen, by means of the magnifying-glass, octaëdral crystals. It is remarkable that the arborisation is always directed from the negative pole towards the positive pole, whatever is the position of the two poles, and it is consequently always established in the track of the electrical current. The vegetation of a metal, with the assistance of electricity, seems to imitate, in some measure, that of the natural plants, which constantly incline towards the light, disengaging oxygen by coming in contact with the solar rays.

VIII. When the metallic tree has extended within a short distance of the positive pole its increase is stopped, because its foliage, being infinitely slender in every respect, annihiJates the electrical action by exercising the power of an infinity of points. It even seems, that by the too near approach of the poles, each may acquire the electric fluid from the other; for the extremities of the metallic ramifications have sometimes begun to oxidate while deoxidation was manifested in the positive pole. It is probable that always when the extremities of the two conductor wires become very slender and very nearly approach each other in the water, the gases coming from their decomposition are mixed one with another. Here we have, if I dm not mistaken, an analogy between the decomposition of water by an electrical machine, and that which is effected by the pile of Volta*.

IX. When the current of Galvanic electricity acts upon water either pure or when charged with some soluble substance, the positive pole attracts the oxygenating principle, while the negative pole attracts the oxygenated principle of the liquid. If the proportion of the components of the latter is variable, it becomes oxygenated at the extremity of the wire in communication with the disk of zinc, and deoxygenated at the extremity of the wire in contact with the disk of copper. The following are the proofs of this fact :

- Wollaston, upon decomposing water by the electricity of an ordinary machine, constantly observed that the oxygen and the hydrogen were disen't gaged both at once, while the action of the pile inclines them to show themsetves separately. 
$X$. The muriatic acid becomes so much oxygenated at the positive pole that it acquires the faculty of dissolving the gold coming from the extremity of the conductor wire. The sulphuric and the nitric acids becomo transparent, and appear to be so surcharged with oxygen in the part surrounding that pole, that $I$ think they are capable of producing effects, when in this state, with which we are not as yet acquainted *. At the negative pole the muriatic disengages a good deal of gas $\uparrow$, the sulphuric acid sends forth a strong sulphureous smell by depositing sulphur, and the nitric acid becomes the nitrous by assuming a blue colour. If the position of the two poles is afterwards changed, so that the one occupies the place of the other, every particle of the acid returns, by little and little, to its primitive state, and the effects recommence.

XI. A solution of muriate of tin, traversed by the Galvanic current, precipitates, by little and little, a white powder, coming from the positive pole. This precipitate, redissolved in the muriatic acid and then tried with corrosive sublimate, altered the latter into white, whereas the liquid which had surrounded the negative pole altered it into black. The muriate of tin had thus become more oxygenated at the extremity of the wire, which excited the liberation of the oxygen.

XII. . After a long action of the Galvanic electricity upon the sulphate of iron in solution, the latter becomes turbid, assuming a red colour in the part which surrounded the positive pole. We may ascertain that it then contains a strongly oxidated sulphate of oxygenated iron by trying it with the prussiate of potash, which immediately produces a very fine Prussian blue with this part of the liquid, whereas that which surrounds the negative pole only produces with the same prussiate a precipitate of a greenish white colour.

XIII. The molybdic acid dissolved in the concentrated

- At this degree of oxygenation the enlphuric acid seems susceptible of dissolving gold ; at least, that which I mace use of in this experiment assumed a yellow colour in proportion as it dissolved the extremity of the gold wire which liberated the oxygen. On pouring into this sulphate of gold a solution of green sulphate of iron, a precipitate was formed like the sulphuret of gold.

+ It would be interesting to examine if this gas comes parily from the decompositicn of the arid.

sulphuric 
sulphuric acid, assumes, in the cold, a fine blue colour, which always disappears when heat is applied to the solution. Upon exposing it to the action of the pile of Volta, the vitreous fluid acts in a manner analogous to heat, while the resinous fluid produces an effect analogous to cold; on the positive pole the liquor becomes by degrees perfectly transparent, and the molybdic acid is partly precipitated in the form of a white powder, whereas round the negative pole it always acquires a deeper and dirtier colour. Upon afterwards changing the position of the two poles, so that the one occupies the place of the other, the contrary happens; the transparent part returns to the blue colour, and the blue part becomes transparent.

XIV. When the Galvanic current exercises its influence for a long time upon the solution of an earthy salt, the base of the latter is gradually precipitated round the extremity of the wire of negative electricity. These precipitates, in my opinion, are not the effect of a decomposition by the alkali which is generated at this point in an infinitely small quantity; but I presume that the acid of the salt is there destroyed, or well decomposed, whence it results that its earthy base becomes free.

The glass tubes containing the solutions submitted to the experiments I have here described, were often covered with a metallic crust, which seems as if melted upon the vitreous matter of the interior of the tube, and which comes from the particles of metal detached by the action of the apparatus of the conductor wires: thus when these extremities were of gold or silver, the glass tubes became perfectly gilded either with the one or the other metal.

Chap. II.

Theory of the Decomposition of Liquids by means of Galvanic Electricity.

XV. The decomposition of water by the electrometer apparatus has for a long time exercised the ingenuity of chemists and naturalists, to whom this phænomenon affords a delicate problem to resolve in order to reconcile it with the theory of the nature of water. It is first necessary to know 
if the two products of the two Galvanic poles come from one and the same molecule of water, or rather from two different molecules; and in the latter case we may ask what becomes of the hydrogen at the place where oxygen only is perceived? and in return, what becomes of the uxygen where hydrogen only is perceived ?

XVI. The column of Volta, which will immortalize his name, is an electrical magnet, every elcment of which (i.e. each pair of disks) possesses its nemative and positive pole. The consideration of this polarity suggested to me the idea that it might establish a similar polarity among the elementary molecules of the water solicited by the same electrical agent; and I confess that this afforded me a spark of light on the subject.

XVII. Let us suppose, therefore, that at the moment of the generation of the hydrogen and the oxygen, there takes place in these two bodies, as well by contact as by the friction of the one against the other, a separation of their natural electricity in such a manner that the former acquires the positive and the latter the negative state; it follows, that the pole from which the resinous electricity continually flows will attract the hydrogen by rejecting the oxygen, whereas the pole animated with the vitreous electricity will attract the oxygen by rejecting the hydrogen *. Thus, when the Galvanic current traverses a quantity of water, each of the two component principles of the latter is solicited by an attractive force and by a repulsive force, of which the centres of action are reciprocally opposite, and which, by acting in the same manner, determines the decomposition of this liquid.

XVIII. The action of each force, in respect to a molecule of water situated in the direction of the Galvanic current, is in the inverse ratio of the square of the distance to which it exercises its influence. But as the distance of any given molecule placed between the two centres of action can never

- Considering the diversity of substances deposited at the negative pois, it would be more simple, and perhaps more just, to admit only an attractive and repulsive fotce acting upon the oxygen, without attributiag it to the poles in relation to the hydrogen.

diminisk 
diminish relatively to the one, without inereasing itself on account of this diminution relatixely to the other, so each of the two elements of such a molecule is selicited by a constant force. which results from the attractive and the repulsive force *.

The effect of the repulsion, although effectively existing. is not sensible, on account of the reciprocal action of the elementary molecules in contact, whence results a recombination of such as are repelled by the Galvanic poles.

XIX. Let us consider, howeyer, a cerlain quantity of water, composed of oxygen, represented hy the negative sign (-), and of hydrogen marked by the positive sign ( + ). See + fig. 1. Plate IX. At the moment of establishing a current of Galvanic electricity in this water, the electrical polarity manifests itself among its elementary molecules in such a manner, that the latter seem to constitute the complement of the pile in action. At the same time all the molecules of oxygen situated in the track of the current will haye a tendency to make their way towards the positive pole, whereas all the molecules of hydrogen situated in the same track will tend towards the negative pole.

It thence results, that when the molecule of water represented by $o, h$, yields its oxygen $o$ to the vitreous fluid of the wire + , its hydrogen $h$ is immediately reoxygenated by the arrival of another molecule of oxygen $o$, the hydrogen of which, $h$, is recombined with $r$, and so on. The same thing takes place, but in a contrary sense, relatively to the molecule of water $Q, P$, which, on yielding its hydragen $Q$, to the resinous fuid of the - wire, is immediately rehydrogenated by the arrival of the molecule $X$; and this succession of decomposition and of recombination of the elements of the water will continue until the latter is completely decomposed.

XX. It is clear that in the whole operation the molecules of water, situated at the extremities of the conductor wires, will alone be decomposed, whereas all those placed interme-

- I suppose that each force has the same intensity; a circumstance which ought actually to take place, since neither of the poles of the electrometer apparatus can acquire electricity except at the expense of the other.

diately 
mediately will change reciprocally and alternatively their component principles without changing their nature. From this I infer, that if it were possible to establish a current of Galvanic electricity in water, in such a manner as to describe in the latter a perfectly circular line, all the molecules of the liquid situated in this circle would be decomposed and instantly recomposed: whence it follows, that this water, although undergoing the effect of the Galvanic action, will always remain water.

XXI. Having exposed some liquids, contained in two or more vases, to the action of the electrometer apparatus, I perceived the polarity at the extremities of the metallic wires which serve to establish the communication between the liquids inclosed in each vase. (See fig. 2.) Thus, when the vases contained acetate of lead dissolved in water, I obtained oxygen at the extremities $a$ and $c$, while the vegetations already described rose at the extremities $b$ and $d *$.

Upon bringing the vessels nearer together, and upon curtailing the dimensions of the wire $l c$ as much as possible, the electrical polarity was nevertheless distinctly perceptible; by imagining the same wire infinitely small, one may conceive how the molecules $n$ and $p$ unite upon regenerating the body which was at first decomposed.

XXII. The theory of the decomposition of water here given leads us to the following consequences:

(a) The proportion of hydrogen could not have increased in the part of the water which is nearest to the positive pole, since the oxygen of the whole quantity of the liquid trayersed by the Galvanic current inclines lowards this point, while the hydrogen endeavours to recede from it.

(b) An oxygenation in that part of the water which surrounds the negative pole is equally impossible, since the hydrogen is there constantly attracted, while the oxygen is repelled from it. See $\S I X$.

* I have communicated my menoir to M. Morichini. This chemist informed me that he obtained an analogous result upon examining the gases which are liberated when the gases only contain water. The extremities $a$ and $c$ yielded oxygen gas, while the hydrogen gas came from the extremiries $b$ and $d$.

Vol. 25. No. 100. Sept. 1806. Z $\quad$ (c) When 
(c) When even the component principles of water are not susceptible of any other proportion of combination than of that which makes it water, the latter would not be less decomposed in the manner described; but there would neither be oxygenation, nor hydrogenation, nor acidity, nor alkalinity, in any part.

XXIII. The production of an acid at the positive pole, and that of an alkali at the negative*, urged by Galvanic electricity, is also a support to the theory proposed; for, according to analogy, we ought to attribute the former to an oxygenation, and the other to the presence of hydrogen $t$. See $\S I X$.

My apparatus having remained several days in action, the cloths moistened with a solution of muriate of soda were here and there covered with a saline efflorescence, which was nothing else than soda united to carbonic acid which it had absorbed from the air.

XXIV. The polar arrangement, such as exists in the elementary molecules of water traversed by the Galvanic current, ought to be established equally among the elementary molecules of every other liquid body, provided they are solicited by the same forces. In the metallic solutions the eleetric polarity takes place among the elements of the oxide, the oxygen of which passes to the positive pole, and the metal of it is deposited at the negative pole. The acid reacts upon these metallic particles which it holds in solution; but being decomposed, as well by this re-action as by the electrical power, the revival does not the less take place.

$\mathrm{XXV}$. I filled a bent tube with two different metallic soIutions, in such a manner that each of them, without being mixed with the other, occupied one of the moieties of the

* Tincture of turnsole, traversed by the Galvanic current, becomes red around the positive pole, and returns to the blue colour upon changing the respective position of the two poles; but these effects may be cxplained by the action of the oxygen and the hydrogen at the moment of their production upon the colouring matter, and are not sufficient to deduce from it the acidity and alkalinity.

t Hydrogen is a constituent part of volatile alkali, and oxygen enters into the composition of all the acids with the nature of which we are acqualnted. 
tube, and they had a simple point of contact in the middle*. On exposing the two liquids thus arranged to the action of the Galvanic current, and on plunging the negative pole borh into the one and the other, it was always covered with revived metal in whatever solution it was plunged.

If we knew any other substance besides oxygen which may be acted upon by the positive pole, we might repeat this experiment relatixely to the latter. An analogous result would then evidently prove that the decomposition of water by Galvanic electricity takes place in relation to two different molecules; an opinion generally admitted, and conformable to the theory which I have now submitted to the examination of the learned.

The admirable simplicity of the law to which this phænomenon is submitted, coincides, to our astonishment, with the laws of the universe. Nature can neither create nor destray; since the number of bodies is never augmented nor diminished, but all without exception are subject to a mutual exchange of their elements: and when we consider the wonderful effects of electricity, which acts often in secret, although spread over the universe, we cannot refrain from pronouncing it to be one of the most powerful agents of the grand operations of Nature.

LII. On the Imitation of Marlle and Plaster Figures ly a new Composition made of old Paper reduced to Pastet.

$J_{\text {ohn Nicholas Gardeur, an artist of Paris, has invented }}$ the method of imitating the most beautiful sculptures by means of old paper reduced to paste; it is also ascertained that this new species of ornament adds, to a wonderful lightness and solidity, the requisite truth in the expression of the figures.

M. Gardeur is the first who attempted, with success, this new branch of industry; and almost all the theatres and

- We may easily perform this operation if we make use of two sulutions of different colours; for instance acetate of lead and nitrate of copper.

f From Billioth. Phys. Econ, for December 1805. 